\title{
A Tutorial on Sentence Semantics Using Lambek Pregroup Grammar and Categorical Quantum Protocols
}

\author{
Prof. M. Adeeb Ghonaimy \\ Professor Emeritus, Faculty of Engineering. \\ Ain Shams University, Cairo, Egypt \\ adeebghonaimy@gmail.com \\ adeeb.ghonaimy@eng.asu.edu.eg
}

\begin{abstract}
Sentence semantics depends mainly on two basic principles: the principle of compositionality [Partee et al, 1990] (sometimes called Frege's principle), and the distributional principle. Briefly, the compositionality principle states that the meaning of a complex expression is a function of the meaning of the parts and the syntactic rules by which they are combined. The distributional principle is that words that occur in a similar context tend to have similar meaning [Turney and Pentel, 2010].

In this tutorial, the syntax used in compositionality is Lambek pregroup grammar [Lambek, 2006]. In order to integrate the above concepts together, categorical quantum protocols were used [Abramsky, and Coecke; 2004] to develop a categorical compositional distributional model of meaning [Grefenstette and Sadrzadeh, 2011][Coecke, et al, 2010] [Kartsaktis, 2014]. This model is sometimes abbreviated as DisCoCat model. This tutorial gives outline for this model explaining the basic elements of the principles involved including Lambek pregroup grammar and categorical quantum protocols.
\end{abstract}

\section{INTRODUCTION}

Sentence semantics depends on two basic principles: the compositionality principle [Partee et al., 1990] and the distributional principle [Turney and Pentel, 2010]. The first one is attributed to Frege'sprinciple that the meaning of a sentence is a function of the meaning of its parts. The second is related to Wittgenstein's philosophy of "meaning in use", where meanings of words can be determined from their context. In 2010, [Coecke et al, 2010] used high-level concepts from categorical quantum protocols to combine compositional and distributional models, the grammar used is Lambek's pregroup grammar [Lambek, 2008] [Lambek, 2006]. An introduction to categories is given by [Coecke and Paquette, 2011]. This combined model is abbreviated as DisCoCat [Grefenstette, and Sadrzadeh, 2011].

In order to give an idea about this model, a number of topics will be presented in the following sections. Section 2 will deal with Lambek pregroup grammar with some definitions and simple examples, section 3 will deal with Categorical Quantum Protocols [Abramsky and Coecke, 2004] with definitions of Category Theory. Section 4 will deal with compositional and distributional models of meaning. Section 5 discusses the unification of compositional distributional categorical models of meaning (the DisCoCat model) together with experimental support for it [Grefenstette and Sadrzadeh, 2011]. Section 6 is the conclusion.

\section{LAMBEK PREGROUP GRAMMAR}

Let us first define the pregroup. A pregroup is a partially ordered monoid (a semigroup with unity element). Each element $a$ has a left adjointalanda right adjointa ${ }^{r}$ such that

$$
\mathbf{a}^{l} \mathbf{a} \rightarrow \mathbf{1} \rightarrow \mathbf{a a}^{l}, \quad \mathbf{a a}^{r} \rightarrow \mathbf{1} \rightarrow \mathbf{a}^{r} \mathbf{a}
$$

Here the arrow denotes partial order. A relation that is reflexive, antisymmetric, and transitive is called a partial order [ Epp,1993].

i. e., for all $\mathrm{a}, \mathrm{b}$, and $\mathrm{c}$, in $\mathrm{P}$ where $\mathrm{P}$ is a set and that $\leq$ is a relation on $\mathrm{P}$, we have that.

$\mathrm{a} \leq \mathrm{a}$ (reflexivity)

if $\mathrm{a} \leq \mathrm{b}$ and $\mathrm{b} \leq \mathrm{a}$ then $\mathrm{a}=\mathrm{b}$ (antisymmetry)

if $\mathrm{a} \leq \mathrm{b}$ and $\mathrm{b} \leq \mathrm{c}$ then $\mathrm{a} \leq \mathrm{c}$ (transitivity).

A set with a partial order on it is called a partially ordered set, poset. Lambek considered Free Pregroups and posets of basic types, which may differ from one language to another, and which is meant to express certain elementary 
grammatical concepts. From the basic types one forms simple types by repeated adjunction. Thus a simple type has one of the following forms:

$$
\ldots a^{l l}, a^{l}, a, a^{r}, a^{r r}, \ldots
$$

where a is a basic type. A compound type is a string of basic types. The types form a monoid under concatenation (1 being the empty string). The partially ordered monoid of types is a pregroup with adjunctions defined inductively thus:

$$
1^{l}=1=1^{r},(\mathrm{xy})^{l}=\mathrm{y}^{l} \mathrm{x}^{l},(\mathrm{xy})^{r}=\mathrm{y}^{r} \mathrm{x}^{r}
$$

The resulting pregroup is the free pregroup generated by the given poset of basic types.

Let us now consider the pregroup of types freely generated by a poset of basic types for a small fragment of English.

$\pi_{\mathrm{j}}=\mathrm{j}^{\mathrm{th}}$ personal subject pronoun, where $\mathrm{j}=1, \ldots, 6$ denotes the three persons singular followed by the three persons plural. In modern English, the original second person singular has disappeared and was replaced by the second person plural. Moreover, there is no morphological distinction between the three plural verb forms.

$\mathrm{s}_{\mathrm{k}}=$ declarative sentence in the $k$ th simple tense $(\mathrm{k}=1,2)$ where they stand for the present and past indicative respectively.

$\mathrm{q}_{\mathrm{k}}=$ yes-or-no questions in the $k^{\text {th }}$ simple tense.

$\mathrm{o}=$ direct object.

$\mathrm{p}_{2}=$ past participle of intransitive verb.

$\mathrm{i}=$ infinitive of intransitive verb.

Both of the last-mentioned types may also apply to compound verb phrases.

$\pi=$ subject when the person is irrelevant.

$\mathbf{q}=$ yes-or-no question when the tense is irrelevant.

Let us now consider a small fragment of English:

He has type $\pi_{3}(=$ third person subject)

Her has type o (=direct object)

Sees has type $\pi_{3}^{r} \mathbf{s}_{1} \mathbf{o}^{l}$ to indicate that we require a third person subject on the left and a direct object on the right.

Now look at the sentence

he sees her

$$
\pi_{j}\left(\pi_{3}^{r} \mathbf{s}_{1} \mathbf{0}^{l}\right) \mathbf{0} \rightarrow \mathbf{s}_{1}
$$

We calculate in two steps

$$
\begin{aligned}
& \boldsymbol{\pi}_{3}\left(\pi_{3}^{r} \mathbf{s}_{1} \mathbf{o}^{l}\right)=\left(\boldsymbol{\pi}_{3} \pi^{\mathbf{r}}\right) \mathbf{s}_{1} \mathbf{o}_{l^{-} \rightarrow} \mathbf{1} \mathbf{s}_{1} \mathbf{o}^{l}=\mathbf{s}_{1} \mathbf{o}^{l} \\
& \left(\mathbf{s}_{1} \mathbf{0}^{l}\right) \mathbf{0}=\mathbf{s}_{1}\left(\mathbf{o}^{l} \mathbf{0}\right) \rightarrow \mathbf{s}_{1} 1=\mathbf{s}_{1} .
\end{aligned}
$$

It is convenient to indicate contraction by underlines.

Similarly, we have 


\section{$\boldsymbol{\pi}_{1}\left(\pi^{r} \mathbf{s}_{2} \mathbf{0}^{l}\right) \mathrm{o}-\rightarrow \mathbf{s}_{2}$}

where the first underline represents the generalized contraction

$$
\pi_{1} \pi^{r} \rightarrow \pi \pi^{r} \rightarrow 1
$$

In the next example we make use of two further type assignments:

$$
\begin{gathered}
\text { Has has type } \pi_{3}^{r} \mathbf{s}_{1} \mathbf{p}_{2}^{l} \\
\text { Seen has type } \mathbf{p}_{2} \mathbf{o}^{l}
\end{gathered}
$$

The former requires one complement on each side, the latter only a simple complement on the right to give

He has seen her

$$
\underbrace{\pi_{3}\left(\pi_{3}^{r}\right.} \mathbf{s} \mathbf{\underbrace} \underbrace{\left.\mathbf{p}_{2}^{l}\right)\left(\mathbf{p}_{2} \mathbf{o}^{l}\right) \mathbf{o}} \rightarrow \mathbf{s}_{1}
$$

Note in contrast

I have seen her

$$
\underbrace{\pi_{1}(\pi_{1}^{r} \mathbf{s}_{1} \underbrace{l}_{2})\left(\mathbf{p}_{2} \mathbf{o}^{l}\right) \mathbf{o}} \rightarrow \mathbf{s}_{1}
$$

You had seen her

$$
\boldsymbol{\pi}_{2}(\pi_{2}^{r} \mathbf{s}_{2} \underbrace{l}_{2})\left(\mathbf{p}_{2} \mathbf{0}^{l}\right) \mathbf{0} \rightarrow \mathbf{s}_{2}
$$

Unfortunately, has must be assigned a different type in direct questions, namely

$$
\text { Has: } \mathbf{q}_{1} \mathbf{p}_{2}^{l} \boldsymbol{\pi}_{3}^{l}
$$

To obtain

$$
\text { Has he seen her? }
$$

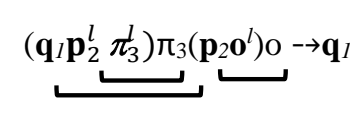

In Lambek's book a detailed presentation of English grammar is given including:

Nouns, adjectives, verbs, adverbs.

Negative and interrogative sentences

Indirect questions.

Doubly transitive verbs.

He gave also a list of the posets of basic types.

It should be noted finally that there are aspects of the English language that were not considered. I give here one example "the irregular verbs" in which Steven Pinker considered in his book Words and Rules [Pinker, 1999].

Regarding other languages, Pregroups have been used to analyze the sentence structure of many languages, for example, French, German, Italian, Polish, Arabic, Japanese, and Persian. Therefore, it is possible to use it to study comparative structures of different languages. 


\section{CATEGORICAL QUANTUM PROTOCOLS}

The tools available for developing quantum algorithms and protocols until 2004 were low-level. However it was learned from Computer Science the importance of compositionality, types, and abstractions [Abramsky and Coecke, 2004, 2005, 2008]. A simple exposition was given by [Coecke, 2005] with an exposition for Categories given by [Coecke and Paquette, 2010]. In this tutorial, I am going to use the simple exposition given by Coecke.

Let us start by defining a category. Consider a system of type $A$ and perform an operation $f$ on it. Then, we have,

$$
\mathrm{A} \stackrel{f}{\longrightarrow} \mathrm{B}
$$

where A is the initial type of the system, B is the resulting type and $f$ is the operation. One can also perform an operation

$$
\mathrm{B} \stackrel{g}{\longrightarrow} \mathrm{C}
$$

and write $g$ o $f$ for the consecutive application of these two operations. Clearly we have

$$
(h \circ g) \circ f=h \circ(g \circ f)
$$

If we further set

$$
\mathrm{A} \stackrel{{ }^{1} A}{\longrightarrow} \mathrm{A}
$$

For the operation "do nothing on a system of type A" we have

1 B of $=$ f $\mathbf{~ o ~} \mathbf{1}_{A}=\mathbf{f}$

Hence, we can define a Category $\mathrm{C}$ as consisting of:

- $\quad$ Objects A, B, C, ...

- $\quad$ Morphisms $f, g, h, \ldots \in \mathrm{C}(\mathrm{A}, \mathrm{B})$ for each pair A, B.

- Associative composition, i. e

$f \in \mathrm{C}(\mathrm{A}, \mathrm{B}), g \in \mathrm{C}(\mathrm{B}, \mathrm{C}) \Rightarrow g$ o $f \in \mathrm{C}(\mathrm{A}, \mathrm{C})$

with $(b \circ g)$ of $=h \circ(g \circ f)$

- An identity morphism $1_{\mathrm{A}} \in \mathrm{C}(\mathrm{A}, \mathrm{A})$ for each $\mathrm{A}$, i. e.

- $f$ o $l_{A}=1_{\mathrm{B}}$ of $f=f$

When in addition we want to be able to conceive two systems $\mathrm{A}$ and $\mathrm{B}$ as one whole $\mathrm{A} \otimes \mathrm{B}$ and also to consider compound operations $f \otimes g$ : $\mathrm{A} \otimes \mathrm{B} \rightarrow \mathrm{C} \otimes \mathrm{D}$, then we pass from ordinary categories to a (2- dimensional) variant called monoidal categories.

Let us now consider what is called the language of pictures which has some primitive data (lines, boxes, triangles, and diamonds) in which we have two kinds of composition, namely parallel (conceiving two systems as a compound single one) and sequential (concatenation in time) and which will obey a certain axiom. Then we derive some results using this picture calculus, e. g. teleportation, logic gate teleportation and entanglement swapping

The primitive data of our formalism consists of:

(1) Boxes with an input and an output which we call "operation" or "channel"

(2) Triangles with only an output which we call "state" or "preparation", procedures" or "ket"

(3) Triangles with only an input which we call "co-state" or "measurement branch"or "bra"

(4) Diamonds without inputs or output, which we call "values" or "probabilities" or "weights"

(5) Lines which might carry a symbol to which we refer as the "type" or the "kind of system, and the A-labeled line itself will be conceived as "doing nothing to a system of type A" or the "identity of A"

Figure (1) shows the primitives of the language of pictures. 


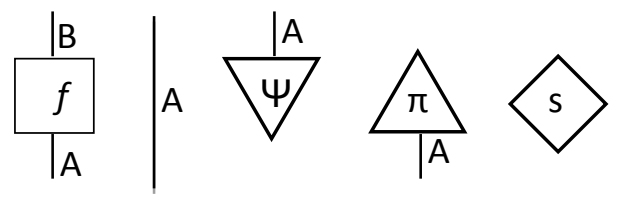

Figure (1): Primitives of the language of pictures

Figure (2) shows examples of combinations of different picture primitives.

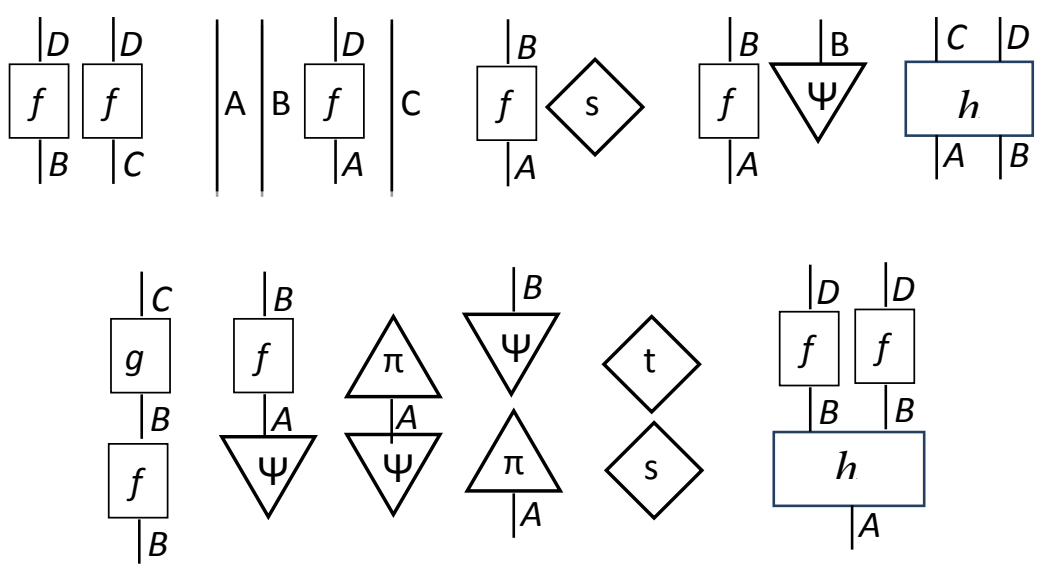

Figure (2): Examples of combinations of different picture primitives

If we connect up a state and costate (i. e. we produce a bra-ket) we obtain a diamond shape since no inputs nor outputs remain. Thus we obtain what we called probability. On the other hand if we connect up a costate and a state (i. e. produce a ket-bra) we obtain a square shape with a genuine input and a genuine output.

Fig. (3) and Fig. (4) show also some useful identities.

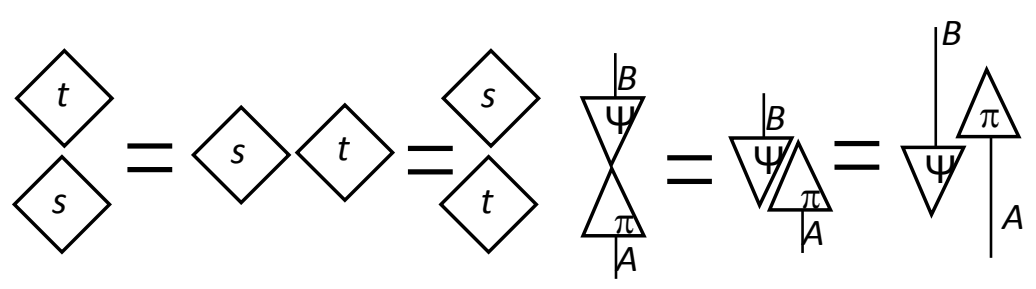

Figure (3): Some useful identities

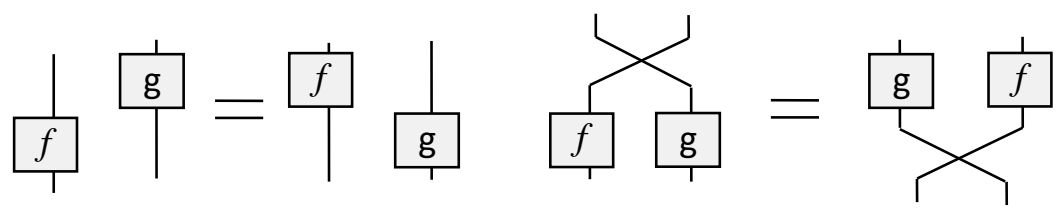

Figure (4): Some more identities

It is also assumed that lines carry an orientation which means that there exists an operation on types which sends each type $A$ to a type $\mathbf{A}^{*}$ with the opposite orientation. We refer to $\mathbf{A}^{*}$ as A's dual. We also assume that for each box $f: A \rightarrow B$ there exists one upside down box $f^{t}: B \rightarrow A$ called $f^{\prime \prime}$ s adjoint. These situations are shown in fig. (5). 

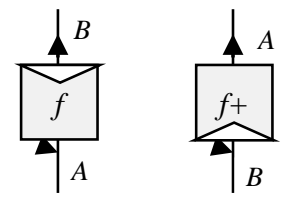

Figure (5): Dual and Adjoints

\section{COPOSITIONAL AND DISTRIBUTIONAL SEMANTICS}

Let us first give a brief idea about vector-based models of word meaning. One of the early methods was gained from the field of information retrieval [Salton, G. and McGill, M. J., 1984].

Also, a more modern exposition is given by [Van Rijsbergen, 2004] which concentrates on the geometry of information retrieval and gives an introduction of its relation to quantum mechanics. The idea of Vector Space Models (VSM) is to represent each document as a point in a space (a vector in a vector space).

Points that are close together in this space are semantically similar and points that are far apart are semantically distant. VSM performs well on tasks that involve measuring the similarity of meaning between words, phrases, and documents. They are also related to the distributional hypothesis which means that words that occur in similar contexts tend to have similar meaning. In order to apply this abstract hypothesis leads to vectors, matrices, and higher order tensors [Turney, P. D. and Pantel, P. 2010].

The principle of compositionality is the principle that states that the meaning of a complex expression is a function of the meaning of its parts and the way these parts are syntactically combined. A number of researches have tried to reconcile the frameworks of distributional semantics with the principle of compositionality. Let us first consider composition models: [Mitchel and Lapata M., 2008]. Some researchers formulate semantic composition as a function of two vectors u and $\mathbf{v}$. They assume that individual words are represented by vectors acquired from corpus. The word's vector typically represents it co-occurrence with neighboring words. A hypothetical semantic space is illustrated in Fig.(6).

\begin{tabular}{|l|l|l|l|l|l|}
\hline & animal & stable & village & gallop & jokey \\
\hline Horse & 0 & 6 & 2 & 10 & 4 \\
Run & 1 & 8 & 4 & 4 & 0 \\
\hline
\end{tabular}

Figure (6): A hypothetical semantic space for horse and run.

Here, the space has only five dimensions and the matrix cells denote the co-occurrence of the target words (horse and run) with the context words (animal, stable, and so on).

Let $\mathbf{p}$ denote the composition of two vectors $\mathbf{u}$ and $\mathbf{v}$, representing a pair of constituents which stand in some syntactic relation $\boldsymbol{R}$. We can thus define a general class of models for this process of composition as:

$$
\mathbf{p}=f(\mathbf{u}, \mathbf{v}, \boldsymbol{R})
$$

If we consider $\boldsymbol{R}$ is fixed to a single well defined linguistic structure, for example the verb-subject relation, then we can write:

$$
\mathbf{p}=f(\mathbf{u}, \mathbf{v})
$$

This still leaves $f$ unspecified.

If we assume that $\mathbf{p}$ lies in the same space as $\mathbf{u}$ and $\mathbf{v}$, avoiding the issues of dimensionality associated with tensor products, and that $f$ is a linear function, then we generate a class of additive models:

$$
\mathbf{p}=\mathbf{A} \mathbf{u}+\mathbf{B} \mathbf{v}
$$

where $\mathbf{A}$ and $\mathbf{B}$ are matrices which determine the contribution made by $\mathbf{u}$ and $\mathbf{v}$ to produce $\mathbf{p}$.

In contrast, if we assume that $f$ is a linear function of the tensor product of $\mathbf{u}$ and $\mathbf{v}$, then we obtain multiplicative models

$$
\mathbf{p}=\mathrm{C} \mathbf{u} \mathbf{v}
$$


where $\mathrm{C}$ is a tensor of rank 3 which projects the tensor product of $\mathbf{u}$ and $\mathbf{v}$ into the space of $\mathbf{p}$.

Further constraints can be introduced to reduce the free parameters in these models leading finally to:

$$
\mathbf{p}_{\mathrm{i}}=\mathrm{u}_{\mathrm{i}}+\mathrm{v}_{\mathrm{i}}
$$

and

$$
\mathbf{p}_{\mathrm{i}}=\mathrm{u}_{\mathrm{i}} \cdot \mathrm{v}_{\mathrm{i}}
$$

For example, the addition of two vectors representing horse and run in Fig. (6) would yield

$$
\text { Horse }+ \text { run }=\left[\begin{array}{lllll}
1 & 14 & 6 & 14 & 4
\end{array}\right]
$$

whereas their product is given by

$$
\text { Horse.run }=\left[\begin{array}{lllll}
0 & 48 & 8 & 40 & 0
\end{array}\right]
$$

As a result of the assumption of symmetry, both these models are "bag of words" models and word -order insensitive. Relaxing the assumption of symmetry in the case of simple additive model produces a model which weighs the contribution of the two components differently as

$$
p_{i}=\alpha u_{i}+\beta v_{i}
$$

The previous reference contains more details about this approach using for evaluation the British National Corpus (BNC) together with some parsed versions of it.

Another research in this direction was given by [Van de Cruys, T. et al, 2014]. In this paper the authors modeled compositionality as a multi-way interaction between latent factors which are automatically constructed from corpus data. Here, they used the UKWAC corpus which is a 2 billion word corpus automatically harvested from the Web, also together with a parsed version of it. Also, they used a tensor-based factorization model. They obtained better results than the previous paper.

One of the main problems in the previous approach that uses simple addition and multiplication is the commutativity of the operators: they treat the sentence as a "bag of words" where the word order does not matter, for example equating the meaning of the sentence "dog bites man" with that of "man bites dog". This fact motivated researchers to seek solutions based on non-commutative operators, such as the tensor product between vector spaces [Kartsaklis, D. 2014]. Thus the composition of two words is achieved by a structural mixing of the basis vectors that results in an increase of dimensionality:

$$
\vec{w}_{1} \otimes \vec{w}_{2}=\sum_{i, j} c_{i}^{w 1} c_{j}^{w 2}\left(\vec{n}_{i} \otimes \vec{n}_{j}\right)
$$

The meaning of a word is then represented as the tensor product of the word's context vector with another vector that denotes the grammatical relationships. As an example, the meaning of the sentence "dog bites man" is:

$$
\overrightarrow{\text { dog bites man }}=(\overrightarrow{\text { dog }} \otimes \overrightarrow{\text { subj }}) \otimes \overrightarrow{\text { bites }} \otimes(\overrightarrow{\text { man }} \otimes \overrightarrow{o b j})
$$

Thus the bag of word problem is solved at the expense of increasing the dimensionality. This new problem was solved in this paper using some categorical concepts. Other papers tackled also this issue [Clark, S. et al., 2008] and [Coecke, B. et $\mathrm{al}, 2010]$ where the last paper gave the mathematical foundations for the compositional distributional model of meaning.

Due to the role of Categorical Quantum Protocol in the unification of the different models for sentence semantics we devote the following section to research in this direction and finally discuss two papers for evaluating these models.

\section{DISTRIBUTIONAL COMPOSITIONAL CATEGORICAL (DisCoCat) MODEL OF MEANING}

In this section we will see how to combine distributional and compositional semantics together with the axiomatic framework for dealing with quantum information processes [Abramsky, S. and Coecke, B., 2004] [Clark, S. et al, 2013] which admits purely diagrammatic calculus [Coecke, B., 2010]. The teleportation protocol in quantum mechanics [Nielsen, M. A, and Chuang, I. L., 2000] [Benenti, G, et al, 2004] provides a corner stone for the diagrammatic reasoning techniques. In Fig. (7) we show the derivation of the general teleportation protocol where the $f$-label represents both the measurement outcome and the corresponding correction performed by Bob [Coecke, B., 2010]. 


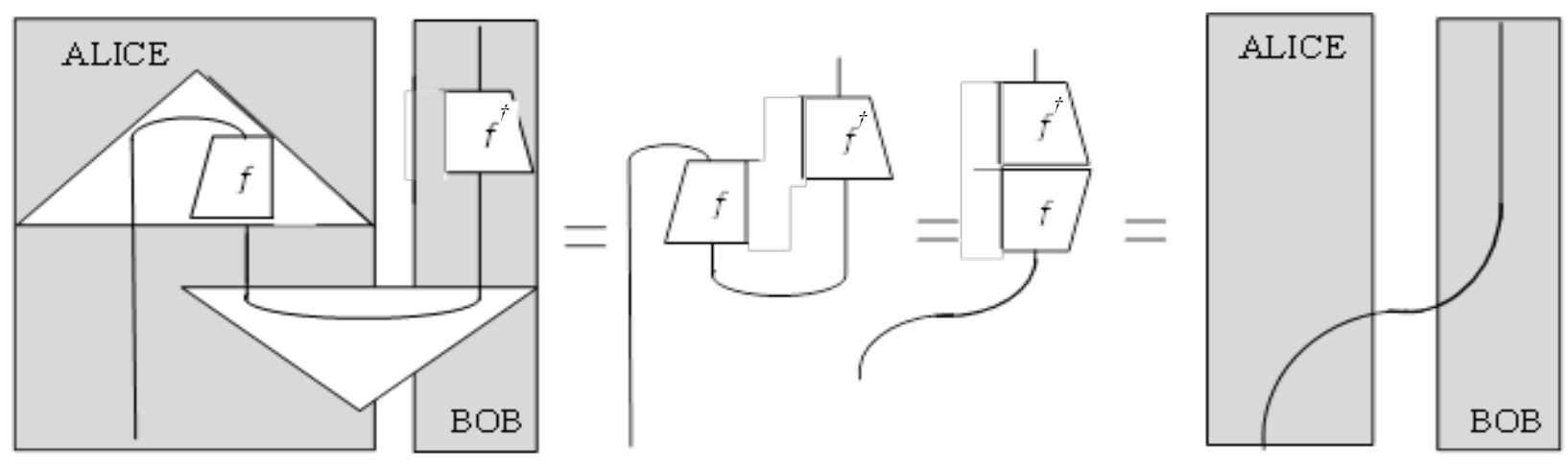

Figure (7): General Teleportation

The main conceptual idea behind these diagrams is that besides these operational physical meaning, they also admit a "logical meaning" in terms of information flow. Referring to Fig. (8), the dashed line represents the logical flow which indicates the state incoming at Alice's side first gets acted upon by an operation $f$, and then by its adjoint $f{ }^{\dagger}$ which in the case that $f$ unitary results in the outgoing state at Bob's side being identical to the incoming state at Alice's side.

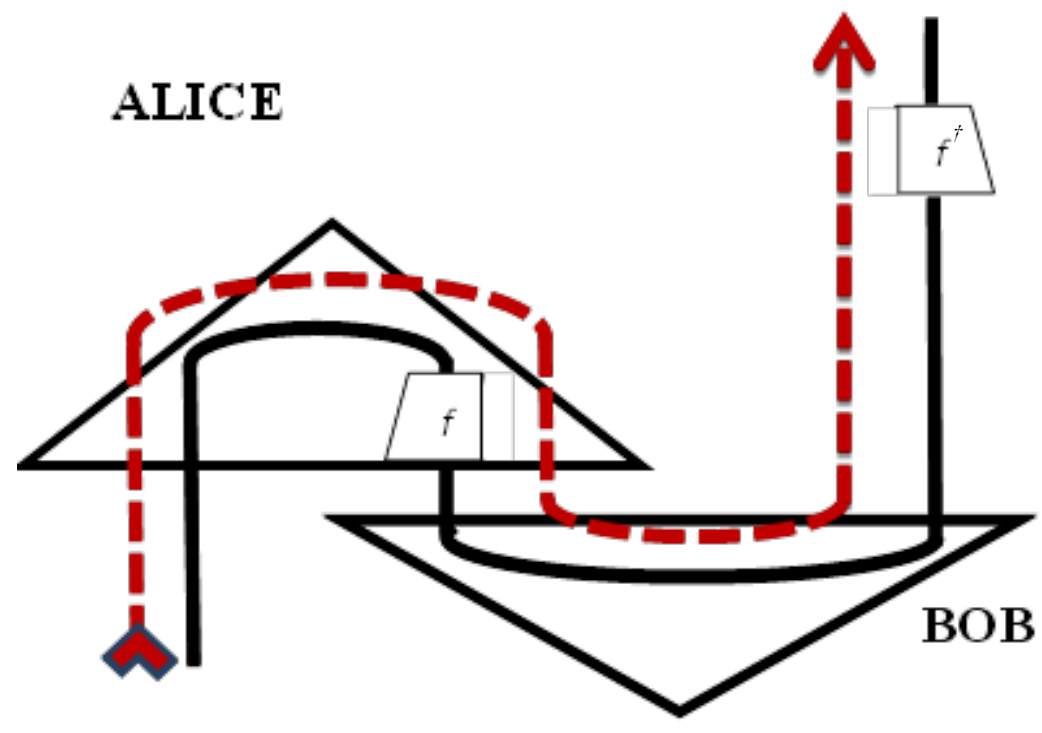

Figure (8): Logical Information Flow and Physical Flow

When interpreted in Hilbert space, the key ingredients of this formalism are "cups" and "Caps":
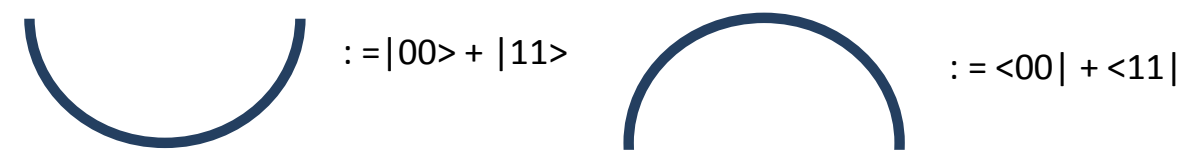

and the equation that governs them is:

$$
\left((<00|+<11|) \otimes \mathbf{I}_{d}\right)\left(\mathbf{I}_{d} \otimes(|00>+| 11>)\right)=\mathbf{I}_{d}
$$

which diagrammatically depicts as:

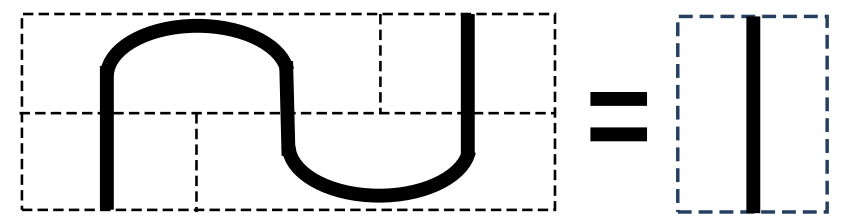


To apply the above concepts to Natural Language Processing, let us consider an example for transitive verbs. A transitive verb requires both an object and a subject to yield a grammatically correct sentence. Consider the sentence "Alice hates Bob": Assume that the words in it are represented by vectors which we denote by triangles:

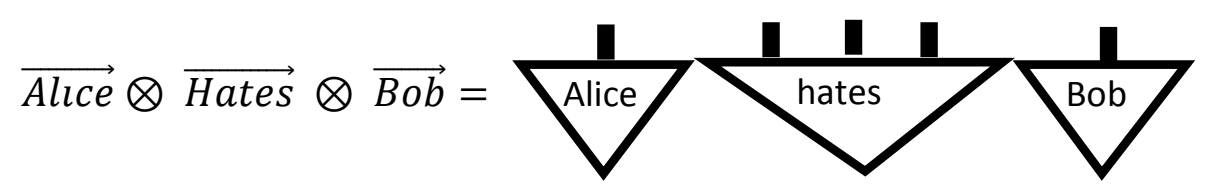

How do these words interact to produce the meaning of the sentence. We feed the meaning of vectors $\overrightarrow{A l i c e}$ and $\overrightarrow{B o b}$ into the verb $\overrightarrow{\text { hates }}$ which then output the meaning of the sentence. Fig. (9) shows how to achieve this

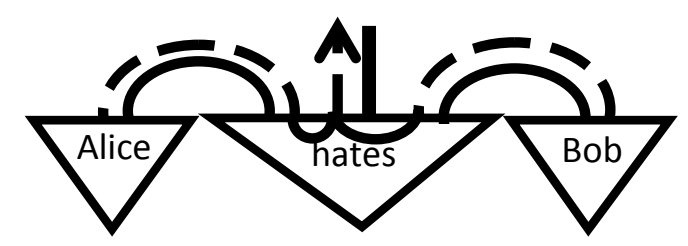

Figure (9): How the transitive verb interacts with the subject and object

Let us see how this example is represented using Lambek pregroup grammar.

Alice hates Bob

$\mathrm{n}$

$$
\begin{array}{r}
\mathrm{n}^{l} \mathrm{sn}^{r} \quad \mathrm{n} \quad=\left(\mathrm{nn}^{l}\right) \mathrm{s}\left(\mathrm{n}^{r} \mathrm{n}\right) \\
\leq 1 \mathrm{~s} 1 \\
=\mathrm{s}
\end{array}
$$

Thus, this is a valid grammatical structure for a sentence

The inequalities using $\mathrm{n}^{l} \mathrm{n} \leq 1$, and $\mathrm{n}^{r} \mathrm{n} \leq 1$ can also be represented with "directed" caps:

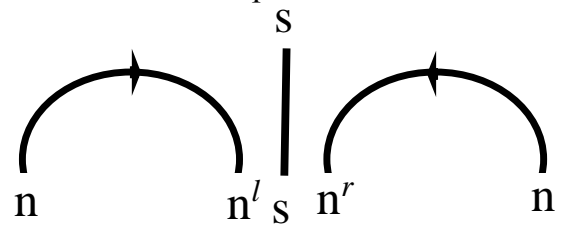

In category theoretic language, both the diagrammatic language for quantum axiomatic and pregroups are called compact closed categories, while the quantum language is symmetric, pregroups have to be non-symmetric given the importance of word order in sentences. As another example, consider the following sentence:

"Alice does not like Bob"

where" does" and "not" are assigned only "logical" meanings.

Fig. (10) Shows the details of this example.

Some authors have given experimental support for this Distributional Compositional Categorical model of meaning (DisCoCat) [Grefenstette, E. and Sadrzadeh, M., (2011) (1)] [Grefenstette, E. and Sadrzadeh, M., (2011) (2)], and indicated that it is a promising approach. However, more experimentation is still needed and this represents only the beginning. 


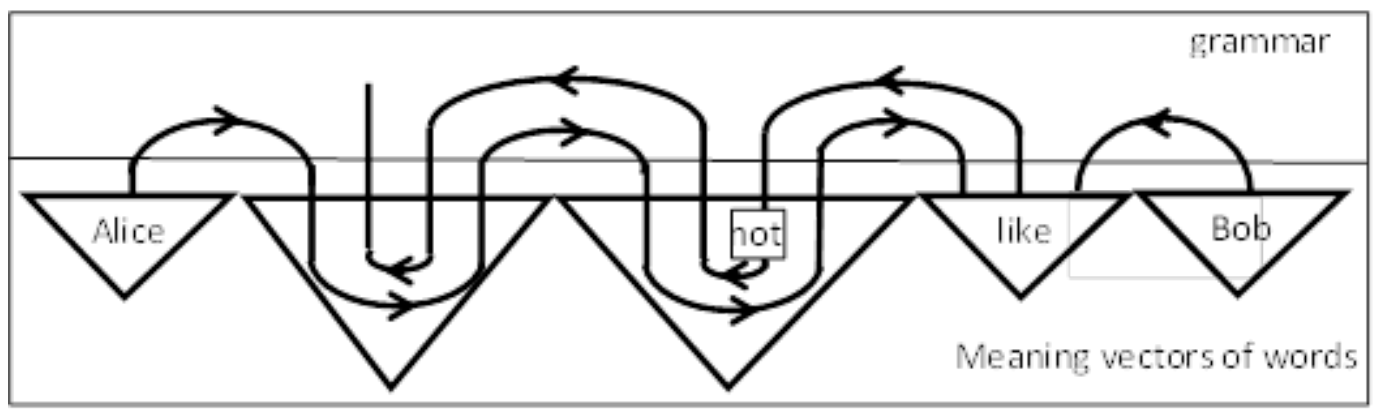

Figure (10): The sentence (Alice does not like Bob)

\section{CONCLUSIONS}

In this paper the different basic models for sentence semantics were presented and then how to unify them together. The first model was the compositional model which needs a grammar that has to be checked first to make sure the grammaticality of the sentence. The grammar chosen was Lambek pregroup grammar. This choice is related to the categorical model for describing the high level quantum protocol that is needed for unifying the different semantic models.

The second model was the distributional semantic model which is an empirical model and needs a large corpora. The corpora considered were the British National Corpus and another one harvested from the Web, and is called UKWAC.

The unification model was called DisCoCat, where the name reflects that this model unifies the Distributional Compositional Categorical models together. Although the grammar used is for the English language, Lambek pregroup grammar could be used for other languages. A reference was given that compared the grammars for a number of languages, among them the Arabic language.

It should be noted that high-level quantum models started to appear in the literature in 2004 and its application for linguistics started to appear in 2010. Since then, intensive applications in linguistics attracted a large number of researchers.

\section{REFERENCES}

[1] Abramsky, S. and Coecke, B. "A Categorical Semantics of Quantum Protocols", Proc. Of the $19^{\text {th }}$ Annual IEEE Symposium on Logic in Computer Science, PP. 415 - 425. IEEE Computer Science Press, 2004.

[2] Benenti, G, et al "Principles of Quantum Computational and Information" World Science, 2004,

[3] British National Corpus, Wikipedia, 30/10/2015.

[4] Clark, S, et al. "A Quantum Teleportation Inspired Algorithm Produces Sentence Meaning From Word Meaning and Grammatical Structure". ArXiv: 1305.0556.Oct. 2013.

[5] Clark, S. et al, "A Compositional Distributional Model of Meaning", Proc. Conf. On Quantum Interactions, University of Oxford, 2004, PP. 133 -140.

[6] Cocke, B. and Paquette, E. O. "Categories for the Practicing physicist, In Coecke, B. editor, "New structures for physics", Lecture Notes in Physics, PP. 167 - 271. Springer, 2010.ArXiv: 0905.3010.

[7] Coecke, B. et al, "Mathematical Foundation for a Compositional Distributional Model of Meaning". Linguistic Analysis 36, Lambek et al, 2010.

[8] Coecke, B. Quantum Picturism. "Contemporary Physics 51, 59 -83. ArXiv; 0908.1787.

[9] Coecke, B. "Kindergarten Quantum Mechanics". Lecture Notes. In Khrennikov, editor, Quantum Theory Reconsideration of the Foundations III, PP. 81 - 98. AIP Press, 2005 ArXiv: quant-ph 0510032.

[10] Epp, S. "Discrete Mathematics with Applications "PWS Publishing Company, 1993.

[11] Ferraresi A, et al. "Introducing and Evaluating UKWAC, a Very large Web - derived Corpus of English", 2008.

[12] Grefenstette, E. and Sadrzadeh, M. "Experimental Support for a Categorical Compositional Distributional Model of Meaning". Proc. Of the 2011 Conference on Empirical Methods of Natural Language Processing. PP. 1394 - 404, Edinburgh, Scotland, U. K. July 2011(1) , Association for Computational Linguistics.

[13] Grefenstette, E. and Sadrzadeh, M. "Experimenting with Transitive Verbs in a DisCoCat" arXiv: 1107, 3119 v2. July 2011(2).

[14] Kartsaklis, P. "Compositional Operators in Distributional Semantics" arXiv:1401. 5327 [cs. CL.]21, Jan. 2014.

[15] Lambek, J. "From Word to Sentence" (A Computational algebraic approach to Grammar". Polimetrico International Scientific Publisher, 2008.

[16] Lambek, J. "Pregroups and Natural Language Processing" The Mathematical Intelligencer, Vol. 28, No. 2, 2006, PP. $41-48$. 
[17] Lambek, J. "The Mathematics of Sentence Structure, "The American Mathematical Monthly, Vol.65, No3, PP.154170, 1958.

[18] Mitchel, J. and Lapata, M. "Vector-based Model of Semantic Composition", Proc. Of ACL- 08: PP. 236 - 244. 2008.

[19] Nielson, M. A. and Chuang, I. L. “Quantum Computation and Quantum Information". Cambridge University press, 2000.

[20] Partee, B. H. et al. "Mathematical Methods in Linguistics", Kluwer Academic Publishers, 1995.

[21] Pinker, S. "Words and Rules." (The Ingredients of Language). Basic Books, 1999.

[22] Sadrzadeh, M. "High Level Quantum Structures in Linguistics and Multi Agent Systems", 2007 American Association for Artificial Intelligence.

[23] Salton, G. and McGill, M. j. "Introduction to Modern Information Retrieval", McGraw - Hill, 1984.

[24] Turney, P. D. and Pantel, P. "From Frequency to meaning: Vector Space Models of Semantics". Journal of Artificial Intelligence Research 37 (2010). PP. 141 -188.

[25] Van de Cruys, T. et al. "A Tensor-based Model of Semantic Compositionality". Conference of the North American Chapter of the Association of Computational Linguistics. May 2014.

[26] Van Rijsbergen, C. J. "The Geometry of Information Retrieval", Cambridge University Press. 2004.

\section{BIOGRAPHY}

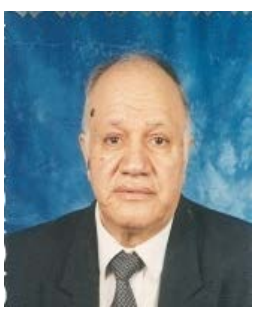

Prof. M. Adeeb Ghonaimy was born on the $28^{\text {th }}$ of December 1936 . He currently holds the position of Professor Emeritus at the Faculty of Engineering, Ain Shams University. In 1999, he was awarded the State Prize of Appreciation in Advanced Engineering Technologies. From 1987 to 1997, he was the Director of the Egyptian Universities Network. From 1979 to 1981 and from 1985 to 1997, he was the Director of the Information Systems Center, Faculty of Engineering, Ain Shams University. From 1979 to 1981 and from 1989 to 1991 he was the Chairman of the Electronics and Computer Department, Faculty of Engineering, Ain Shams University. He got his M.A.Sc and Ph.D degrees from the University of Toronto, Canada in 1961 and 1965 respectively. He got his B.Sc from the Electrical Engineering Department (Communication Section), Faculty of Engineering , Cairo University in 1958.

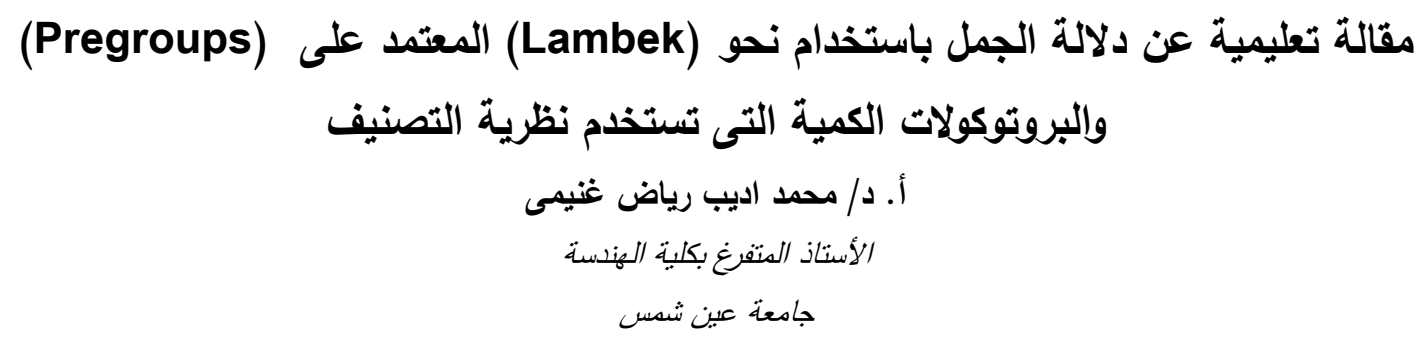

adeebghonaimy@hotmail.com adeeb.ghonaimy@eng.asu.edu.eg

تعتمد دلالة الجملة على نموذجين أساسيين : الأول يسمى نموذج التركيب والثانى يسمى نموذج التوزيع • فكرة التركيب تنص على ان معنى الجملة الكبيرة تكون دالة فى مكونات هذه الجملة التى تربطها قواعد النحو . وفكرة التوزيع تقول أن الكلمات التى توجد فى سياق واحد يكون لها معانى منتابهة • ونظرا لأن فكرة التركيب تعتمد على قواعد النحو التى تربط مكونات الجملة فقد تم اختيار نحو Lambek الذى يعتمد على (Pregroups) ). و طربقة التوزيع تتطلب وجود حصائل (corpora) كبيرة منل British) الذى يحتوى على National Corpus 100 مليون كلمة أو UKWAC الذى تم تحصبله من خلال شبكة الإنترنت عن طربق محتوبات الثبكة المعرفية (Web) ويحتوى على أكثر من 2 بليون كلمة ـ ونظراً لأن لكل نموذج مميزاته وعيوبه فقد إبتدأ فى الأونة الأخيرة (بعد سنة 2010) محاولات دمج النموذجين وذلك عن طريق بروتوكولات كمية تستخدم نظرية التصنيف. وأحد هذه النماذج يتم إختصاره إلى (DisCoCat) لتعكس دمج ثناثة نماذج فى نموذج واحد • وقد تمت بعض وسائل التقييم التى بينت الإضافة التى نتجت عن هذا الدمج 